J. Nonlinear Var. Anal. 2 (2018), No. 3, pp. 269-272

Available online at http://jnva.biemdas.com

https://doi.org/10.23952/jnva.2.2018.3.02

\title{
A NOTE ON RANDOM EQUILIBRIUM POINTS OF TWO MULTIVALUED MAPS
}

\author{
EKATERINA GETMANOVA, VALERI OBUKHOVSKII* \\ Faculty of Physics and Mathematics, Voronezh State Pedagogical University, Voronezh, Russia
}

\begin{abstract}
We present a random version of a theorem on equilibrium points for two parametrized multivalued maps satisfying a joint Caristi type condition.

Keywords. Random equilibrium; Random fixed point; Random multivalued map; Measurable multivalued map; Carathéodory multivalued map.
\end{abstract}

2010 Mathematics Subject Classification. 47H10, 28B20, 47H04, 47H40, $49 \mathrm{~J} 55$.

\section{INTRODUCTION}

Caristi's theorem [3] is one of remarkable fixed point results of nonlinear analysis which finds a number of generalizations and applications; see, e.g., $[1,5,6,7]$ and the references therein. In this paper, we obtain a random version of a theorem on the existence of equilibrium points for two parametrized multivalued maps satisfying a joint Caristi type condition.

\section{PRELIMINARIES}

Let us recall some notions from the theory of multivalued maps (details can be found, for example, in $[2,4,5])$.

Let $X$ and $Y$ be metric spaces. By the symbol $C(Y)[K(Y)]$, we denote the collection of all nonempty closed [respectively, compact] subsets of $Y$. If $Y$ is a normed space, the symbol $C v(Y)[K v(Y)]$ denotes the collection of all nonempty convex closed [respectively, compact] subsets of $Y$.

Definition 2.1. A multivalued map (multimap) $\mathscr{F}: X \rightarrow C(Y)$ is said to be upper semicontinuous (u.s.c.) [lower semicontinuous (l.s.c.)] if for every open [respectively, closed] set $V \subset Y$

$$
\mathscr{F}^{-1}(V)=\{x \in X: \mathscr{F}(x) \subset V\}
$$

is an open [respectivbely, closed] subset of $X$.

Definition 2.2. A multimap $\mathscr{F}: X \rightarrow C(Y)$ is said to be continuous if it is both u.s.c. and 1.s.c.

Let $(\Omega, \Sigma)$ be a measurable space, i.e., a set $\Omega$ equipped with a $\sigma$-algebra $\Sigma$ of its subsets.

Definition 2.3. A multimap $\mathscr{F}: \Omega \rightarrow C(Y)$ is said to be measurable if $\mathscr{F}^{-1}(V) \in \Sigma$ for every open set $V \subset Y$.

${ }^{*}$ Corresponding author.

E-mail addresses: ekaterinagetmanova@bk.ru (E. Getmanova), valerio-ob2000@ mail.ru (V. Obukhovskii).

Received February 20, 2018; Accepted June 11, 2018.

(C)2018 Journal of Nonlinear and Variational Analysis 
In what follows, let $(\Omega, \Sigma, \mu)$ be a locally compact metric space with a Radon measure $\mu$ and $\sigma$-algebra $\Sigma$ of $\mu$-measurable subsets.

Let $X, Y$ be separable metric spaces.

Definition 2.4. A multimap $\mathscr{F}: \Omega \times X \rightarrow C(Y)$ is said to be a random u-multimap [random l-multimap] provided

(i) $\mathscr{F}$ is measurable with respect to a minimal $\sigma$-algebra generated by $\Sigma \times B(X)$, where $B(X)$ is the collection of all Borel subsets of $X$;

(ii) for every $\omega \in \Omega$, the multimap $\mathscr{F}(\omega, \cdot): X \rightarrow C(Y)$ is u.s.c. [respectively, 1.s.c.]

If a multimap $\mathscr{F}: \Omega \times X \rightarrow C(Y)$ satisfies condition $(i)$ and condition

$(i i)^{\prime}$ for every $\omega \in \Omega$, the multimap $\mathscr{F}(\omega, \cdot): X \rightarrow C(Y)$ is continuous

we will call it a random multimap.

Definition 2.5. Let $A \subseteq X$ be a closed set. A measurable map $\xi: \Omega \rightarrow A$ is called a random fixed point of a multimap $\mathscr{F}: \Omega \times A \rightarrow C(X)$ if

$$
\xi(\omega) \in \mathscr{F}(\omega, \xi(\omega))
$$

for all $\omega \in \Omega$.

Proposition 2.1. ([4], Proposition 31.3). Let $\mathscr{F}: \Omega \times A \rightarrow C(X)$ be a random u-multimap such that for every $\omega \in \Omega$ the set of fixed points

$$
\operatorname{Fix} \mathscr{F}(\omega, \cdot)=\{x \in X: x \in \mathscr{F}(\omega, x)\}
$$

is nonempty. Then $\mathscr{F}$ has a random fixed point.

Before formulating the next assertion, let us say that a function $\psi: \Omega \times X \rightarrow(-\infty,+\infty]$ is admissible if for every $\omega \in \Omega$ the function $\psi(\omega, \cdot)$ is proper, i.e., its value is finite at least at one point, bounded below, and lower semicontinuous.

The following result is the direct consequence of the multivalued version of the Caristi fixed point theorem (see $[1,5,6])$ and Proposition 2.1.

Theorem 2.1. Let $(X, d)$ be a complete separable metric space and let $\psi: \Omega \times X \rightarrow(-\infty,+\infty]$ be an admissible function. If $\mathscr{F}: \Omega \times X \rightarrow C(X)$ is a random u-multimap such that for every $\omega \in \Omega$ and $x \in X$ there exists $f \in \mathscr{F}(\omega, x)$ such that

$$
\psi(\omega, f)+d(x, f) \leq \psi(\omega, x)
$$

then $\mathscr{F}$ has a random fixed point.

Definition 2.6. We say that $f: \Omega \times X \rightarrow Y$ is a Carathéodory map provided: $(i)$ the map $f(\omega, \cdot): X \rightarrow Y$ is continuous for every $\omega \in \Omega$; (ii) the map $f(\cdot, x): \Omega \rightarrow Y$ is measurable for each $x \in X$.

Analogously, $\mathscr{F}: \Omega \times X \rightarrow K(Y)$ will be called a Carathéodory multimap if: $(i)$ the multimap $\mathscr{F}(\omega, \cdot): X \rightarrow$ $K(Y)$ is continuous for every $\omega \in \Omega$; (ii) the multimap $\mathscr{F}(\cdot, x): \Omega \rightarrow K(Y)$ is measurable for each $x \in X$.

Let us mention the following properties of the Carathéodory multimaps (see, e.g., [5], Propositions 7.9 and 7.16).

Proposition 2.2. If $\mathscr{F}: \Omega \times X \rightarrow K(Y)$ is a Carathédory multimap, then 
(i) $\mathscr{F}$ is measurable;

(ii) if $X$ is complete, then for every $\varepsilon>0$ there exists a closed subset $\Omega_{\varepsilon} \subseteq \Omega$ such that $\mu\left(\Omega \backslash \Omega_{\varepsilon}\right)<\varepsilon$ and the restriction $\mathscr{F} \mid \Omega_{\Omega_{\varepsilon} \times X}$ is continuous.

The next parametrized version of the Michael continuous selection theorem holds true (see [5], Theorem 7.23).

Proposition 2.3. Let $X$ be a complete separable metric space and let $Y$ be a separable Banach space. Let $\mathscr{F}: \Omega \times X \rightarrow C v(Y)$ be an l-random multimap. Then $\mathscr{F}$ admits a Carathéodory selection, i.e., there exists a Carathéodory map $f: \Omega \times X \rightarrow Y$ such that

$$
f(\omega, x) \in \mathscr{F}(\omega, x), \quad \forall(\omega, x) \in \Omega \times X .
$$

\section{A RANDOM EQUILIBRIUM POINT THEOREM}

Theorem 3.1. Let $X$ be a separable Banach space and let $(Y, d)$ be a complete separable metric space. Let $F: \Omega \times X \rightarrow K(Y)$ be a Carathéodory multimap and let $G: \Omega \times Y \rightarrow C v(X)$ be a random l-multimap. Suppose that $\psi: \Omega \times Y \rightarrow(-\infty,+\infty]$ is an admissible function such that for every $\omega \in \Omega$ and $x \in X$ there exists $f \in F(\omega, x)$ with the property that for each $y \in Y$ satisfying

$$
x \in G(\omega, y)
$$

we have

$$
\psi(\omega, y)+d(y, f) \leq \psi(\omega, y) .
$$

Then there exist measurable maps $x_{\star}: \Omega \rightarrow X$ and $y_{\star}: \Omega \rightarrow Y$ such that

$$
\left\{\begin{array}{l}
x_{\star}(\omega) \in G\left(\omega, y_{\star}(\omega)\right), \\
y_{\star}(\omega) \in F\left(\omega, x_{\star}(\omega)\right)
\end{array}\right.
$$

for all $\omega \in \Omega$.

Proof. According to Proposition 2.3, we take a Carathéodory selection $g: \Omega \times Y \rightarrow X$ of the multimap $G$ :

$$
g(\omega, y) \in G(\omega, y), \quad \forall(\omega, y) \in \Omega \times Y .
$$

Consider the multimap $\widetilde{F}: \Omega \times Y \rightarrow K(Y)$ defined as

$$
\widetilde{F}(\omega, y)=F(\omega, g(\omega, y)) .
$$

Let us show that the multimap $\widetilde{F}$ satisfies the conditions of Theorem 2.1.

First, we demonstrate that $\widetilde{F}$ is a Carathéodory multimap. In fact, the continuity of the multimap $\widetilde{F}(\omega, \cdot)$ for every $\omega \in \Omega$ is obvious. Further, by applying Proposition 2.2 (ii), for a given $\varepsilon>0$, we take a closed subset $\Omega_{\varepsilon} \subseteq \Omega$ such that $\mu\left(\Omega \backslash \Omega_{\varepsilon}\right)<\varepsilon$ and the restrictions of $F$ and $g$ to $\Omega_{\varepsilon} \times Y$ are continuous, but then $\widetilde{F}$ is also continuous on $\Omega_{\varepsilon} \times Y$. Hence $\widetilde{F}(\cdot, y)$ is continuous on $\Omega_{\varepsilon}$ for every $y \in Y$. This means that the multimap $\widetilde{F}(\cdot, y)$ satisfies the Lusin property. Hence (see, e.g., Theorem 19.6 in [4]) it is measurable. According to Proposition 2.2 (i), one sees that the multimap $\widetilde{F}$ is measurable and so it is a random multimap.

Now, we take any $\omega \in \Omega$ and $y \in Y$. By the condition of the theorem there exists $f \in \widetilde{F}(\omega, y)=$ $F(\omega, g(\omega, y))$ such that

$$
\psi(\omega, f)+d(y, f) \leq \psi(\omega, y)
$$


By Theorem 2.1, the multimap $\widetilde{F}$ has a random fixed point $y_{\star}: \Omega \rightarrow Y$, i.e.,

$$
y_{\star}(\omega) \in \widetilde{F}\left(\omega, y_{\star}(\omega)\right)=F\left(\omega, g\left(\omega, y_{\star}(\omega)\right)\right) .
$$

It is clear that $g\left(\omega, y_{\star}(\omega)\right)$ is measurable. Hence it may be taken as the desirable map $x_{\star}(\omega)$.

\section{Acknowledgements}

This study was supported by the Ministry of Education and Science of the Russian Federation (Project No. 1.3464.2017/4.6) and the RFBR grants (Projects No. 17-51-52022, 16-01-00370, 16-01-00386).

\section{REFERENCES}

[1] J.-P. Aubin, Optima and equilibria. An introduction to nonlinear analysis, Graduate Texts in Mathematics, 140. SpringerVerlag, Berlin, 1993.

[2] Y.G. Borisovich, B.D. Gelman, A.D. Myshkis, V. V. Obukhovskii, Introduction to the theory of multivalued maps and differential inclusions, 2nd edition, Librokom, Moscow, 2011 (in Russian).

[3] J. Caristi, Fixed point theorems for mappings satisfying inwardness conditions, Trans. Amer. Math. Soc. 215 (1976), 241-251.

[4] L. Górniewicz, Topological fixed point theory of multivalued mappings, 2nd edition, Topological Fixed Point Theory and Its Applications, 4, Springer, Dordrecht, 2006.

[5] S. Hu, N.S. Papageorgiou, Handbook of multivalued analysis. Vol. I. Theory, Kluwer, Dordrecht, 1997.

[6] N. Mizoguchi, W. Takahashi, Fixed point theorems for multivalued mappings on complete metric spaces, J. Math. Anal. Appl. 141 (1989), 177-188.

[7] A. Petrusel, G. Mot, Multivalued analysis and mathematical economics, House of the Book of Science, Cluj-Napoca, 2004. 